\title{
Autoeficacia de los estudiantes de la carrera de Traducción inglés-español en instituciones de educación superior chilenas
}

\section{Self-efficacy of English-Spanish Translation students in Chilean higher education institutions}

\author{
SOLANGE RAMÍREZ \\ solange.ramirez@pucv.cl \\ Pontificia Universidad Católica de Valparaíso
}

Fecha de recepción: 30 de mayo de 2020

Fecha de aceptación: 25 de noviembre de 2020

Resumen: El presente trabajo corresponde a un estudio exploratorio cuantitativo-cualitativo, de diseño secuencial-explicativo, cuyo objetivo es comprender a nivel a macro y micro la autoeficacia de los estudiantes de la carrera de Traducción inglés-español de instituciones chilenas. Para el logro de dicho objetivo, en una primera fase cuantitativa, se aplicó la Escala de Autoeficacia General a 160 estudiantes entre todas las instituciones de educación superior chilenas que imparten la carrera profesional de traducción inglés-español y, para la fase cualitativa subsecuente, se recurrió a una triangulación de las técnicas de protocolo verbal retrospectivo y entrevista en profundidad a 3 participantes de distintas universidades de la $\mathrm{V}$ región de Chile. Los resultados indican que la Escala de Autoeficacia General, a pesar de su frecuente uso en el campo de los estudios de traducción, presenta muy poca especificidad para medir la autoeficacia en estudiantes de traducción inglés-español. Asimismo, los resultados de la etapa cualitativa indican que los factores que tienen mayor influencia en la autoeficacia de los estudiantes de traducción inglés-español corresponden al manejo disciplinar que estos presentan en las distintas áreas al momento de traducir, al rol que tienen sus docentes y compañeros de curso, y a los distintos estados fisiológicos y emocionales que experimentan.

Palabras clave: Autoeficacia, Estudiantes de traducción, Escala de Autoeficacia General, Traducción, Educación superior.

Abstract: This quantitative-qualitative exploratory study, of sequentialexplanatory design, understands at a macro and micro level self-efficacy of English-Spanish Translation program students of Chilean institutions. In a first quantitative phase, the Escala de Autoeficacia Generalwas applied to 160 
students among all the Chilean higher education institutions that teach the professional English-Spanish translation program, and for the subsequent qualitative phase, a triangulation of the Retrospective verbal protocol techniques and in-depth interview was conducted to 3 participants from different universities in the $\mathrm{V}$ region of Chile. The results indicate that the General Self-Efficacy Scale, despite its frequent use in the field of translation studies, has very little specificity to measure self-efficacy in translation students and, among the factors that have the greatest impact on the selfefficacy of English-Spanish translation students, we can find knowledge in different areas, teachers and classmates' role, and physiological and emotional states.

Keywords: Self-Efficacy, Translation students, General Self-Efficacy Scale, Translation, Higher education.

\section{INTRODUCCIÓN Y OBJETIVOS}

El presente trabajo pretende dar a conocer los resultados de una investigación exploratoria cuyo objetivo general era comprender a nivel a macro y micro la autoeficacia de los estudiantes de traducción inglés-español de las instituciones de educación superior chilenas. Sus objetivos fueron:

- Conocer a través de un análisis exploratorio el nivel de autoeficacia de los estudiantes de la carrera de Traducción inglés-español de las instituciones de educación superior chilenas.

- Conocer la confiabilidad de la Escala de Autoeficacia General aplicada a estudiantes de traducción en Chile.

- Describir los factores que influyen en la autoeficacia de los estudiantes de la carrera de Traducción inglés-español de las instituciones de educación superior chilenas.

La presente investigación se compone de un método de investigación mixto dado que se pretendía lograr una comprensión más completa del fenómeno y parte desde la importancia e influencia de los factores emocionales y afectivos en el proceso de traducción. En cuanto al propósito del presente trabajo, se busca generar aportes teóricos pioneros a la disciplina dado que, hasta ahora, nunca antes se ha estudiado la autoeficacia en estudiantes de traducción en Chile. 


\section{ReFERENTES TEÓRICOS}

\subsection{La traducción y su aspecto emocional}

La Traducción corresponde a un proceso interpretativo y comunicativo que consiste en la reformulación de un texto con los medios de una lengua que se desarrolla en un contexto social y con una finalidad determinada (Hurtado Albir, 2001), en donde la cultura es un componente esencial dado que, en primer lugar, la actividad traductora involucra al menos dos idiomas y el idioma es parte importante de la cultura (Al-Hassan, 2013). Sin embargo, la traducción es una actividad compleja que involucra no solo aspectos culturales, sino también múltiples habilidades, factores afectivos y actitudinales (Laukkanen ,1996; Tirkkonen-Condit y Laukkanen, 1996) y experiencias cognitivas y emocionales (Hansen, 2005; Laukkanen, 1996).

\subsection{La autoeficacia}

La autoeficacia es un elemento fundamental de la teoría cognitivosocial y se define como la medida en que un individuo cree que puede dominar una tarea en particular (Bandura, 1995). Las creencias de autoeficacia funcionan como un conjunto importante de determinantes proximales de la motivación, el afecto y la acción humana, que operan sobre la acción a través de procesos de intervención afectivos y cognitivos (Bandura, 1989). En base a lo anterior, es importante destacar que la autoeficacia no hace referencia a nuestras habilidades y aptitudes reales, sino que a las percepciones que tenemos de estas (Blanco, Martínez, Zueck y Gastelum, 2011; Rice, 1997).

De acuerdo con la teoría cognitivo-social, la creencia que tenga un individuo de sí mismo predecirá mejor la conducta y el éxito futuro que las capacidades que el individuo tenga objetivamente (Bandura, 1995; Salanova Soria et al, 2004; Valiante, 2000). Pues, esto implica que cuando una persona tiene una mayor confianza y creencia de que puede hacer algo, tendrá más posibilidades de conseguirlo (Aragón, 2012; Blanco et al., 2011; Rice, 1997). De la misma forma, las personas con mayor autoeficacia visualizan escenarios de éxito y sienten que las tareas a las que se enfrentan son oportunidades (Bandura, 1989, 1997; Krueger y Dickson, 1994) e interpretan los posibles errores como algo informativo en vez de algo que los debilita (Tabernero, 2004). Sin embargo, los individuos con menor autoeficacia suelen visualizar escenarios de fracaso y perciben riesgos en las tareas que deben enfrentar (Bandura, 1989, 1997; Krueger y Dickson, 1994). 


\subsubsection{Fuentes de autoeficacia}

De acuerdo a la teoría cognitivo-social, las personas generan, a través de su capacidad de autorreflexión, sus creencias de autoeficacia a partir de cuatro fuentes principales (Bandura, 1986, 1987, 1997): experiencias directas, aprendizaje vicario, persuasión verbal y estados fisiológicos y emocionales (Bandura, 1994, 1997).

\subsubsection{Experiencias directas}

En primer lugar, las experiencias directas, también denominadas experiencias de dominio y logros de ejecución (Woolfolk, 2014), corresponden a cómo el individuo percibe las experiencias pasadas de fracasos y éxitos en situaciones similares y son la fuente más importante de autoeficacia (Peinado, 2010) porque manifiestan si se tienen o no realmente las capacidades necesarias para realizar una tarea (Bandura, 1997) ya que el éxito incrementa la autoeficacia y los fracasos las disminuyen (Bandura, 1997; Pajares, 1997; Woolfolk, 2014).

\subsubsection{Aprendizaje vicario}

El aprendizaje vicario es proporcionado por modelos sociales y hace referencia a las sensaciones que los individuos perciben a través de otras personas, específicamente, cuando ven que personas parecidas a ellos logran ciertas metas, se incrementa la creencia de que ellos también podrán tener éxito (Prieto, 2007). Asimismo, si observan que individuos similares a ellos fracasan, disminuye su motivación y la creencia de que podrán lograr sus objetivos (Bandura, 1995; Zeldin y Pajares, 2000). Dentro de las experiencias vicarias, el grado de «similitud» que el individuo siente que tiene con la persona observada es un elemento clave debido a que cuanto mayor sea la similitud asumida, más persuasivos serán los éxitos y fracasos de los modelos de conducta observados (Bandura, 1995).

Si bien esta fuente es menos influyente que las experiencias de dominio, es muy relevante cuando el individuo no siente seguridad con respecto a sus capacidades o cuando ha tenido muy pocas experiencias directas respecto a una tarea (Canto y Rodríguez, 1998).

\subsubsection{Persuasión verbal}

La persuasión verbal se refiere los comentarios provenientes de aquellas personas del entorno acerca de nuestras capacidades, los cuales, en forma de retroalimentación, pueden reforzar o disminuir la autoeficacia (Prieto, 2007; Zalazar, Aparicio, Ramírez, Garrido, 2011). La efectividad de la persuasión verbal está mediada por factores como la estructura de la retroalimentación, la experticia y credibilidad de la persona que nos trata de 
persuadir y, además, que tan digna de confianza nos parezca esa persona (Maddux, 1995; Torre, 2007).

Dada su naturaleza de retroalimentación, tanto oral como escrita, es la estrategia utilizada con mayor frecuencia dentro del ámbito educacional para desarrollar la autoeficacia en los estudiantes (Torre, 2007).

\subsubsection{Estados fisiológicos y emocionales}

La cuarta fuente de autoeficacia corresponde a los estados fisiológicos y emocionales. Los sentimientos negativos como nerviosismo, cansancio, estrés, y estados de ánimo positivos (Zalazar et al., 2011), que las personas experimentan durante la ejecución de una tarea o actividad (Bandura, 1997) actúan como activadores positivos o negativos de la autoeficacia, lo cual resulta en el sentimiento de estar o no «mentalmente preparado» (Woolfolk, 2014). Dicho de otra forma, el aumento de las emociones fisiológicas y emocionales de bienestar refuerzan la autoeficacia y los estados emocionales negativos la disminuyen (Usher y Pajares, 2009).

\subsection{Autoeficacia en la traducción}

En cualquier área de la actividad humana, las suposiciones de los individuos sobre sus propias capacidades para realizar ciertas tareas de manera apropiada juegan un papel importante en el resultado final (Schwarzer, 1992) y la traducción no es una excepción. Así pues, la autoconfianza, los componentes afectivos y actitudinales conforman los pilares fundamentales dentro de varios modelos que describen a la competencia traductora (Atkinson, 2012; Grupo PACTE, 2001; GonzalezDavies y Scott-Tennet, 2005; Göpferich, 2009; Kelly, 2002; Pym 1991, 1992).

En la actividad traslativa, la autoeficacia se define como la confianza que el traductor tiene para traducir (Haro-Soler, 2017). A pesar de que hace apenas una década empezó a utilizarse el término de "autoeficacia» en Traducción (Haro-Soler, 2018a), de que son muy pocos los estudios de la autoeficacia en la traducción que se han publicado hasta ahora (BolañosMedina, 2014; Albin, 2012) y de que las investigaciones acerca de las habilidades emocionales en la competencia traductora siguen siendo «embrionarias», tal como lo destacan los expertos en competencia (Collombat, 2016), todos coinciden en que la importancia de la autoeficacia radica en que la percepción del éxito, que influye en la eficacia real, en el rendimiento y la capacidad de realizar la actividad profesional de traducción. Específicamente, la autoeficacia interviene en la etapa de organización y de dirección de las acciones. Dependiendo de su nivel en un dominio dado, la autoeficacia puede hacer que el traductor comience o evite una tarea. Además, en la etapa posterior a dicha tarea, la autoeficacia determina si, ante 
el fracaso, el traductor persiste en la tarea, o qué cantidad de esfuerzo está dispuesto a dedicarle (Albin, 2012).

1.3.1 Actividades específicas de la traducción influenciadas por la autoeficacia

En cuanto a las actividades propias de la Traducción que se ven afectadas por la autoeficacia, en primer lugar, y retomando las palabras de Albin (2012), podemos encontrar la toma de decisiones. Un ejemplo de esto podría ser la elaboración de soluciones ante problemas de traducción. Pues según, Nuñez y Bolaños-Medina (2017) la autoeficacia de los estudiantes de traducción para resolver problemas de traducción se correlaciona positivamente con su motivación intrínseca. Asimismo, en un estudio desarrollado por Kussmaul (1995), se descubrió que los traductores descartaban soluciones adecuadas debido a su inseguridad. Lo anterior también coincide con Lorenzo $(1999,2002)$, quien sugiere que la falta de autoeficacia corresponde a uno de los problemas principales para los traductores al momento de evaluar sus propias decisiones tanto durante el proceso de traducción como en el producto final.

En segundo lugar, podemos encontrar el uso de herramientas de traducción. Específicamente, el tener una gran autoeficacia y un estilo favorable de atribución está claramente relacionado un alto nivel de gestión y de capacidad para trabajar con herramientas TAO (Albin, 2012) y diccionarios bilingües (Fraser, 1995).

Por su parte, Hansen (2003) y Tirkkonen-Condit y Laukkanen (1996) señalan que la confianza y la calidad de la traducción parecen estar relacionadas de manera positiva: si las circunstancias aumentan la sensación de seguridad del traductor, es más probable que asuma el papel de un comunicador en lugar de un simple procesador de textos. Por ende, los programas de formación universitaria en Traducción, al ser una etapa tan influyente en el futuro desempeño profesional de los traductores, deben diseñarse para brindarles a los estudiantes la oportunidad de desarrollar una autoimagen profesional positiva, así como la confianza en su trabajo (Tirkkonen-Condit y Laukkanen, 1996).

Bolaños-Medina (2014), en una investigación preliminar y exploratoria acerca de la autoeficacia de estudiantes de Traducción en español y alemán, concluyó que la autoeficacia está estrechamente relacionada con la comprensión de lectura en lengua de origen, las habilidades de documentación y con el hecho de saber cuándo dejar de buscar una solución para un problema de traducción. Lo anterior tiene estrecha relación en lo descubierto en el estudio de Think-Aloud-Protocol realizado por Araghian, Ghonsooly y Ghanizadeh (2018): los traductores que tienen menos confianza 
en sus capacidades se demoran más tiempo en terminar las traducciones dado que sienten la necesidad constante de volver a revisar lo que han hecho.

Asimismo, tanto Bolaños-Medina $(2014,2015)$ como Fraser (1995) concluyeron que la autoeficacia tiene influencia en la tolerancia a la ambigüedad que se puede presentar en el texto de origen al momento de traducir.

Otra actividad propia de la traducción relacionada con la autoeficacia es la pericia, entendida como un rendimiento consistentemente superior que se muestra al realizar ciertas tareas específicas del dominio (Ericsson y Charness, 1997; Ho, 2010; Muñoz-Martin, 2014). Concretamente, la pericia en traducción implica las capacidades que subyacen en el desempeño de traductores humanos expertos, incluido el amplio conocimiento de dominio, pero también reglas heurísticas que simplifican y mejoran los enfoques de resolución de problemas, metaconocimiento y metacognición (Muñoz-Martín, 2009).

Por su parte, Kussmaul (1995) postula que la confianza que uno tiene en sí mismo corresponde a un prerrequisito para la traducción creativa, la cual tiene que ver con el uso impredecible no institucionalizado del lenguaje (Wilss, 1996) o la selección de una variante de traducción que no está regida por reglas (Alexieva, 1990). En palabras más simples, la traducción creativa corresponde a aquella traducción que se escapa de la estructura de la traducción literal, la cual quedó demostrada hace siglos que es demasiado «forzada» y que alguna forma de reescritura es inevitable (Ballard, 1997). Lo anterior está en consonancia con lo propuesto por Froeliger (2004), quien sugiere que la falta de autoeficacia puede provocar que los traductores se atengan demasiado al texto de origen, incluso si es que se han percatados de errores en el mismo y, por ende, realizar una traducción más literal que creativa.

La autoeficacia también se correlaciona en gran medida con la motivación en la carrera, pues cuando se trata de este aspecto, la falta de autoeficacia puede actuar como una limitación o restricción. Además, la autoeficacia se correlaciona de forma positiva con el éxito laboral, pues tiende a predecir la satisfacción laboral, el número de encargos recibidos por semana y el rango de ingresos (Atkinson, 2012). Dentro de este mismo ámbito laboral, los traductores con alta autoeficacia se evalúan a sí mismos atendiendo a criterios de dinero y prestigio, mientras que los traductores con baja autoeficacia son más autorreferentes (Albin, 2012). 


\subsection{La autoeficacia en la formación de traductores}

En un contexto pedagógico, fomentar la autoeficacia en los estudiantes constituye un factor clave dado que tiene una influencia beneficiosa no solo a corto plazo, sino también a través de su desarrollo académico y profesional (Bandura, 1986). Puesto que la autoeficacia afecta la motivación y el aprendizaje de los alumnos (Bandura, 1997) dado que los estudiantes con altos niveles de autoeficacia tienen mayor motivación, obtienen mejores resultados académicos, regulan su aprendizaje de forma más eficaz y tienen mayor motivación intrínseca para aprender que otros estudiantes (González y Tourón, 1992).

La disciplina de la Traducción no está exenta de todo esto dado que la autoeficacia es una competencia más que se espera de todo traductor (HaroSoler, 2017) y si se puede desarrollar mientras los estudiantes aún están en su proceso de formación, y se encuentran dentro del ambiente relativamente «protector» de su institución de educación superior, existe la ventaja de permitirles enfrentar desafíos de forma constructiva y, además, pueden obtener guía de forma relativamente fácil por parte de sus profesores y compañeros de curso (Bolaños-Medina y Núñez, 2018). En cuanto a los factores que pueden influir en la autoeficacia, un estudio de enfoque metodológico mixto desarrollado por Haro-Soler (2019), se concluyó que, el trabajo colaborativo entre los estudiantes influye de forma muy positiva en la autoeficacia de los estudiantes y, que también pueden influir de forma favorable los docentes, el aprendizaje vicario a través de exalumnos de la misma carrera, y el currículum, siempre y cuando este último este bien construido (Haro-Soler, 2018b)

Tal como expresa Haro-Soler (2017), a pesar de lo relevante que es la autoeficacia en la formación de traductores, hoy existe un vacío en el desarrollo de autoeficacia en estudiantes de Traducción (Atkinson y Creeze, 2014), el cual, según Presas (1998) y Way (2014), puede ser producto de lo difícil que es plasmar competencias relacionadas con la psicología del traductor en objetivos didácticos. Sin embargo, esa ausencia en el desarrollo de autoeficacia no solo es identificada por investigadores académicos y docentes, sino también por los mismos estudiantes (Haro-Soler, 2017).

\subsection{La carrera de Traducción inglés-español en Chile}

Para contextualizar, cabe destacar que el sistema de educación superior chileno se compone de cuatro tipos de instituciones: universidades, institutos profesionales, centro de formación técnica e instituciones de educaciones superior de las Fuerzas Armadas y la Policía. Asimismo, las instituciones pueden ser clasificadas si es que son privadas o pertenecientes al consejo de rectores (Bernasconi, 2004). 
En Chile, las instituciones de educación superior pueden someterse voluntariamente al proceso de acreditación institucional, en el cual se evalúa el cumplimiento de su proyecto corporativo y verificar la existencia de mecanismos eficaces de autorregulación y de aseguramiento de la calidad, así como propender al fortalecimiento de su capacidad de autorregulación y al mejoramiento continuo (Comisión Nacional de Acreditación, 2020).

Actualmente, la carrera profesional de traducción inglés-español se imparte en nueve instituciones en Chile y corresponde a una de las carreras con peor empleabilidad y más bajos ingresos económicos y, además, la industria nacional de la traducción no goza de prestigio debido a que es una labor desvalorizada y no reconocida; lo cual resulta en un mercado laboral muy reducido y poco rentable (Word Factor, 2015; Hunter, 2015).

\section{METOdOLOGía Y PROCEDIMIENTOS DE APLICACIÓN}

La presente investigación corresponde a un estudio de alcance exploratorio (Naghi, 2000), que se enmarca dentro de un método mixto, el cual se desarrolló en dos fases: una cuantitativa y otra cualitativa (Denzin y Lincoln, 2002; Hamui-Sutton, 2013).

Para la fase cuantitativa, se aplicó la Escala de Autoeficacia General (Scholz, Gutiérrez, Sud y Schwarzer, 2002). Se optó por este instrumento dado que, al momento de realizar esta fase de la investigación, aún no estaba lista la escala desarrollada por Haro-Soler (2018a) «La Escala de Creencias de Autoeficacia del Traductor» y, hasta la fecha, aún no está validada en Chile. Asimismo, la Escala de Autoeficacia General corresponde a una de las más utilizadas en Traducción (Bolaños-Medina y Nuñez, 2018) (por ejemplo, ha sido utilizada en Mashady, Fatollahi y Pourgalavi, 2015; Bolaños-Medina, 2014; Jiménez, Ruiz y Pinazo, 2014) y, además, se procuraba generar un aporte teórico y sentar un precedente dado que nunca antes se había aplicado en estudiantes de traducción en Chile.

Se consideró como población los estudiantes de segundo ciclo (segundo año, tercer año y tesistas) de todas las instituciones de educación superior chilenas que imparten la carrera profesional de Traducción inglésespañol. 


\begin{tabular}{|c|c|c|c|}
\hline Institución & Duración & $\begin{array}{r}\text { Acreditació } \\
\mathbf{n}\end{array}$ & Tipo \\
\hline $\begin{array}{l}\text { Instituto Profesional } \\
\text { EATRI }\end{array}$ & 8 semestres & $\begin{array}{c}\text { No } \\
\text { acreditado }\end{array}$ & Privado \\
\hline $\begin{array}{l}\text { Pontificia } \\
\text { Universidad } \\
\text { Católica de } \\
\text { Valparaíso }\end{array}$ & 9 semestres & 7 años & $\begin{array}{l}\text { Perteneciente al } \\
\text { consejo de rectores }\end{array}$ \\
\hline $\begin{array}{l}\text { Universidad } \\
\text { UNIACC }\end{array}$ & 10 semestres & 3 años & Privada \\
\hline $\begin{array}{l}\text { Universidad } \\
\text { Católica de } \\
\text { Temuco }\end{array}$ & 10 semestres & 3 años & $\begin{array}{r}\text { Pertenecient } \\
\text { e al consejo de } \\
\text { rectores }\end{array}$ \\
\hline $\begin{array}{l}\text { Universidad de } \\
\text { Atacama }\end{array}$ & 10 semestres & $\begin{array}{r}\text { No } \\
\text { acreditada }\end{array}$ & $\begin{array}{r}\text { Pertenecient } \\
\text { e al consejo de } \\
\text { rectores }\end{array}$ \\
\hline $\begin{array}{l}\text { Universidad de la } \\
\text { Serena }\end{array}$ & 8 semestres & 4 años & $\begin{array}{r}\text { Pertenecient } \\
\text { e al consejo de } \\
\text { rectores }\end{array}$ \\
\hline $\begin{array}{l}\text { Universidad de las } \\
\text { Américas }\end{array}$ & 10 semestres & $\begin{array}{r}\text { No } \\
\text { acreditada }\end{array}$ & Privada \\
\hline $\begin{array}{l}\text { Universidad de } \\
\text { Playa Ancha }\end{array}$ & 10 semestres & 4 años & $\begin{array}{r}\text { Pertenecient } \\
\text { e al consejo de } \\
\text { rectores }\end{array}$ \\
\hline $\begin{array}{l}\text { Universidad de } \\
\text { Tarapacá }\end{array}$ & 10 semestre & 3 años & $\begin{array}{r}\text { Pertenecient } \\
\text { e al consejo de } \\
\text { rectores }\end{array}$ \\
\hline
\end{tabular}

Tabla 1: Caracterización Instituciones Participantes en la Investigación Fuente: Elaboración propia

En cuanto a la muestra numérica, se consideró que cada institución tiene en promedio 30 estudiantes de segundo ciclo y, por ende, el universo del presente estudio corresponde a 270 estudiantes. Se calculó la muestra con un margen de error del $5 \%$ y nivel de confiabilidad de un $95 \%$, lo cual arrojó una muestra de 159 individuos. 
Para la fase cualitativa, se recurrió a una triangulación de las técnicas de protocolo verbal retrospectivo y entrevista en profundidad (todas fueron llevas a cabo por la investigadora del presente estudio). Por motivos geográficos, la selección de participantes se concentró en la quinta región de Chile y en total se consideraron tres participantes (uno por cada universidad que imparte la carrera). Dentro de las características se encuentran de una estudiante de una universidad privada tradicional perteneciente al consejo de rectores acreditada por siete años, un estudiante de una universidad estatal pública perteneciente al consejo de rectores acreditada por cuatro años $y$, finalmente, un estudiante de una universidad privada donde la carrera de traducción no estaba acreditada.

El día de la aplicación se citó a los participantes en sus respectivas instituciones educacionales. Se comenzó con la aplicación del protocolo verbal retrospectivo y se les asignó un texto (Anexo 1) para que tradujeran. Específicamente, se utilizó el reporte descriptivo de una biopsia oncológica dado que su alta complejidad terminológica implicaría habilidades y actividades propias de la traducción, como documentación, búsqueda terminológica, descarte de fuentes confiables y no confiables, uso de diccionarios bilingües, uso de herramientas TAO, entre otras. Se les solicitó a los participantes que tradujeran solo la sección de «Hallazgos» (específicamente, 102 palabras), no obstante, se les facilitó la biopsia completa por temas de contextualización. Cabe destacar que todos los participantes firmaron consentimientos informados que permitían grabar en formato de audio la aplicación tanto del protocolo verbal como de la entrevista y también resguardan la identidad y anonimato de los participantes. Asimismo, los estudiantes firmaron otro consentimiento para grabar la pantalla del computador por medio del programa Screen Recorder Lite mientras traducían.

No se les impuso tiempo límite para terminar de traducir, sino que se les dio la libertad de demorarse todo lo que necesitaran. Una vez que terminaron de traducir, los estudiantes realizaron la fase correspondiente a expresarse en voz alta acerca de su proceso de traducción y, además, tenían a su disposición la grabación de la pantalla del computador de todo el proceso de traducción que realizaron en caso de que lo necesitaran para estimular su discurso. Una vez finalizada la aplicación del protocolo verbal retrospectivo, se procedió inmediatamente a la aplicación de la entrevista en profundidad, la cual estuvo inicialmente enfocada en aspectos mencionados por los participantes en la etapa previa de protocolo con el fin de ahondar más en su proceso de traducción. Luego, la entrevista circuló por los ejes temáticos «¿Qué factores influyen para que te sientas más confiado al momento de traducir?», «¿Qué factores influyen para que te sientas menos confiado al 
momento de traducir?» y «¿Para qué áreas disciplinares te sientes más confiado para traducir? ¿Por qué?»

Para propósitos de análisis, las grabaciones de discursos de los participantes emitidos tanto en la etapa de protocolo verbal como en la entrevista fueron transcritas y se procedió a un análisis de contenido. Subsecuentemente, por medio del software Nvivo, se realizó una categorización libre, la cual dio como resultado 199 nodos libres, y luego se realizó la sistematización de los conceptos acerca de los cuales se sustentaría el análisis.

\section{Resultados fase CUANTITATIVA}

En la fase cuantitativa, con respecto a la aplicación de la Escala de Autoeficacia General, los estudiantes de segundo ciclo de la carrera profesional de traducción inglés- español de todas las instituciones de educación superior chilenas presentan un nivel de autoeficacia muy alto. Todas las instituciones educacionales participantes obtuvieron puntajes promedio extremadamente similares, pues el $80 \%$ de las instituciones obtuvo un promedio aproximado de 33 puntos y el $20 \%$ restante obtuvo 32 puntos. Dicha homogeneidad, se puede observar también en la desviación estándar de los resultados dado que fue de 0,4 puntos. Por consiguiente, no era práctico realizar un puntaje de corte en la mediana para clasificar los niveles (alto o bajo) de autoeficacia ni realizar las correlaciones planeadas en un principio.

\section{Resultados FASE CUALITATIVA}

Dada la gran riqueza hallada en los discursos analizados, se realizó la sistematización de los conceptos acerca de los cuales se sustentaría el análisis. El núcleo central fue «Autoeficacia». De este modo y, obedeciendo a lo anteriormente señalado, se elaboraron cuatro matrices correspondientes a:

- Docentes

- Compañeros de curso

- Manejo disciplinar

- Estados fisiológicos y emocionales 


\subsection{Matriz docentes}

La matriz de Docentes (Figura 1) se configura de discursos que aluden a experiencias vicarias y a retroalimentación.

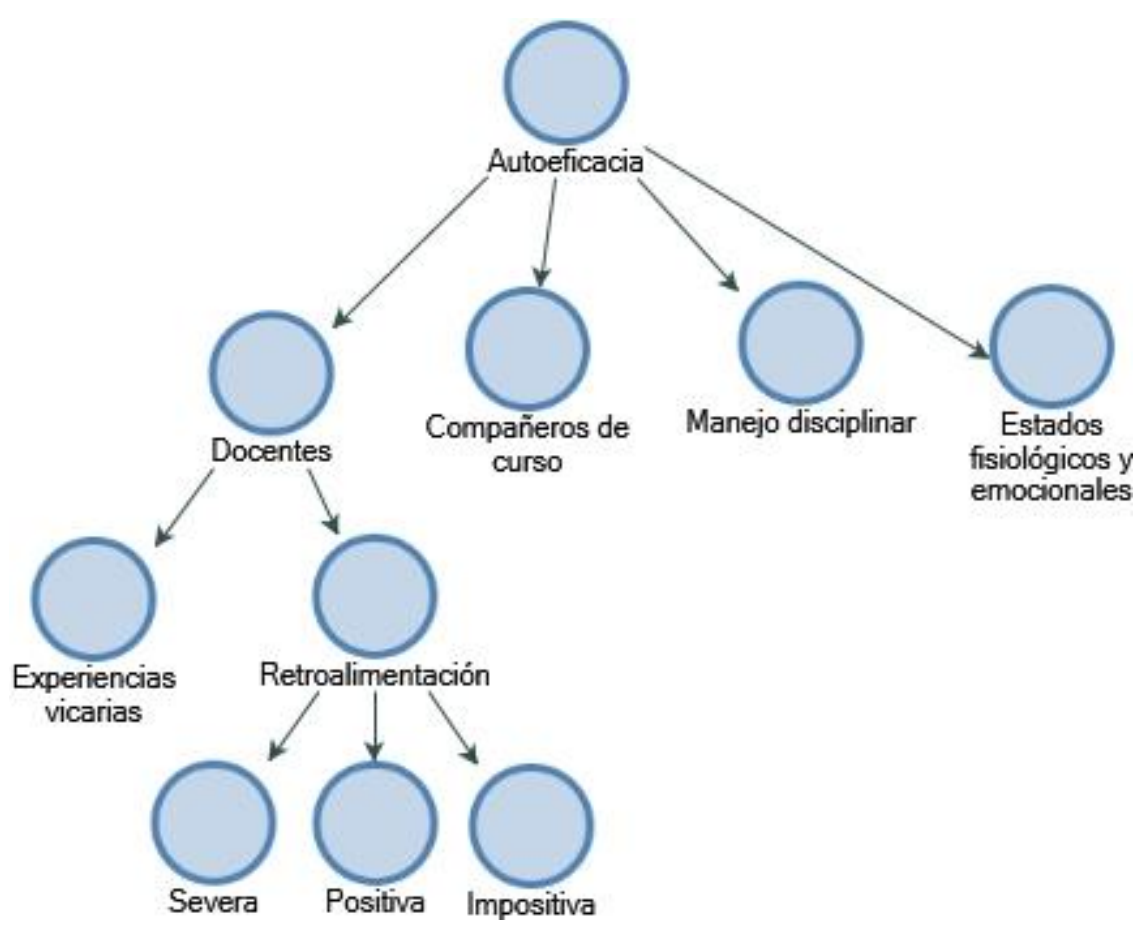

Figura 1. «Matriz Docentes». Fuente: Elaboración propia

\subsubsection{Experiencias vicarias}

Dentro la teoría cognitivo-social, las experiencias vicarias corresponden a una de las fuentes principales de autoeficacia (Bandura, 1995); puesto que, el ver que otros individuos considerados como similares a uno tienen éxito o logran la ejecución de ciertas metas otorga más confianza de que nosotros también podremos hacerlo (Bandura, 1997). Lo particular en los discursos de los entrevistados es que debido a que el "grado de similitud" con los referentes es un factor importante (Bandura, 1995), podría esperarse que surgiera aprendizaje vicario a través de pares directos como compañeros de curso o exalumnos de la universidad (Haro-Soler, 2017, 2019), sin embargo, en este caso se produjo por medio de sus docentes. 
Asimismo, de acuerdo con Canto y Rodríguez (1998), las experiencias vicarias son muy relevantes cuando las personas no han tenido muchas experiencias directas respecto a una tarea o actividad, lo cual en este caso se cumple a cabalidad dado que, todos los entrevistados, al ser estudiantes, aún se encuentran en periodo de formación y, por ende, aún no se enfrentan al mundo laboral o la actividad de traducir profesionalmente en un contexto real.

Lo anterior se ve reflejado en el siguiente discurso emitido por uno de los tres entrevistados, el cual es un ejemplo que alude a experiencias vicarias relacionadas con docentes:

Los vemos como entidades, dentro de las mismas experiencias que nos cuentan, vemos el lado humano. Entonces uno ve que ellos también fueron estudiantes y pasaron los mismos procesos que nosotros, que tuvieron los mismos problemas de rendimiento o confianza, entonces, uno se siente más tranquilo en ese sentido. (Entrevista 1, p. 6)

\subsubsection{Retroalimentación}

Los discursos referidos a retroalimentación hablaban de retroalimentación positiva, retroalimentación severa y retroalimentación impositiva.

\subsubsection{Retroalimentación positiva}

La persuasión verbal como retroalimentación de nuestro desempeño corresponde a una de las cuatro fuentes principales de autoeficacia (Prieto, 2007; Zalazar, Aparicio, Ramírez, Garrido, 2011). Asimismo, corresponde a la estrategia más frecuente en el contexto educacional para desarrollar la autoeficacia en los estudiantes (Torre, 2007) y, si tomamos en consideración que la efectividad de la persuasión verbal está mediada por factores como la experticia y credibilidad de la persona que la emite (Maddux, 1995; Torre, 2007), aquella que está aludida en los discursos emitidos por los participantes es altamente efectiva dado que, como quedó señalado en el apartado anterior, los entrevistados ven a sus profesores como entidades y referentes muy potentes.

Si se lleva al plano específico de la traducción, la retroalimentación positiva y constructiva corresponde a un factor clave para que los estudiantes confíen en sus capacidades como traductores (Araghian et al., 2018). Asimismo, la retroalimentación evidenciada en los discursos de los estudiantes entrevistados tiene dos ventajas, en primer lugar, el hecho de que le expliquen el porqué de sus errores en sus traducciones ayuda a que el alumno comprenda la brecha entre su desempeño y el resultado esperado, lo 
cual, subsecuentemente, les ayuda a autorregular su aprendizaje (Hattie y Timperley, 2007; Nicol y Macfarlane-Dick, 2006; Huxham, 2007; Brookhart, 2008). Asimismo, este aspecto de la retroalimentación en donde se considere el proceso de traducción más que el producto final y, además, se motive a la autorregulación de los estudiantes, refleja el cambio de paradigma que Fox (2000), Gonzalez-Davies y Scott-Tennet (2005), Kiraly (1995) consideran necesario en la enseñanza de la traducción

Lo anterior se ve reflejado en los siguientes discursos acerca de retroalimentación positiva ${ }^{1}$ :

El profesor de repente pone great, awesome, en las traducciones o en una oración que estaba con cierta construcción. Y eso es bueno. (Entrevista 1, p. 7)

Entrevistadora: ¿te pasó alguna vez con un tercero que afectó en tu falta o aumento de confianza?

Entrevistado: Un profesor sí, me decía que tenía buena redacción, que mantenía la fidelidad del mensaje y que iba bien encaminado, me generó mucha confianza. (Entrevista 3, p.10)

\subsubsection{Retroalimentación severa}

En los discursos de los entrevistados se evidenció una situación muy lamentable que actúa en detrimento de la autoeficacia de los estudiantes, la cual aludía al hecho de ser retroalimentados de forma muy severa. Pues, tal como se demostró en el apartado anterior, es importante que, dentro del ámbito de la Traducción, los docentes entreguen una retroalimentación positiva, constructiva, expliquen por qué consideran erróneas las traducciones de sus alumnos, elogien los aciertos, sea orientada al proceso de traducción más que al producto final (Hattie y Timperley, 2007; Nicol y Macfarlane-Dick, 2006; Huxham, 2007; Brookhart, 2008; Fox, 2002; Gonzalez-Davies, 2005; Kiraly, 1995) y sea una retroalimentación exhaustiva que aborde las necesidades específicas de los estudiantes y realmente les ayude a desarrollar sus habilidades de traducción (Pietrzak, 2014). Asimismo, de acuerdo a Bolaños-Medina y Núñez (2018) y Haro-Soler (2018b), el contexto de formación enmarcado por la institución de educación superior debería ser un ambiente protector para generar una retroalimentación constructiva. No el caso contrario, en donde se infunde el miedo y la tensión en los estudiantes.

\footnotetext{
${ }^{1}$ Dos de los tres entrevistados emitieron discursos acerca de retroalimentación positiva y por temas de limitaciones de longitud del artículo se optaron solo por estas dos citas para ejemplificar.
}

Hikma 19 (2) (2020), 291 - 326 
Lo anterior se ve reflejado en los siguientes discursos emitidos por uno de los tres entrevistados acerca de retroalimentación severa²:

Sí, siempre, hay harta preocupación, quizás porque no quieren que la profesora se enoje, no quieren que los reten, pero siempre hay un aire extraño en las horas de revisión. Mucho nerviosismo siempre. (Entrevista 2, p. 11)

Sí, puede pasar, es demasiado sí, al principio la encontré medio intimidante, yo la he visto en otras personas, esa manera de traducir mal no querían seguir traduciendo, traducían completamente mal y se ponían más nerviosos, entonces más mal lo hacían, era un ciclo. (Entrevista 2, p. 9)

\subsubsection{Retroalimentación impositiva}

Algunos discursos de los entrevistados también reflejaron una retroalimentación que no es flexible, en donde no se da espacio para tener más de una versión de la traducción solicitada y donde el docente tiende a imponer su versión de la traducción sobre la de los estudiantes. Esto, según Kussmaul (1995) y Kiraly (1995), es perjudicial para la autoeficacia, dado que el docente, al ser un "guardián de la verdad» y considerar incorrecta toda la traducción que difiera de su versión, merma el desarrollo de la confianza y, según Pym (2003), se pierde la esencia de la traducción: ser un proceso de resolución de problemas donde es necesario que el docente genere un ambiente de aprendizaje que facilite a los estudiantes el exponerse a otras posibles soluciones de los problemas de traducción, no solamente a una única versión correcta, es decir, la del profesor.

Asimismo, específicamente en un caso se evidenció un hecho muy contraproducente en donde la docente exigía que los estudiantes trajeran «las cosas hechas para no discutirlas en clases» dado que, en el ámbito de la Traducción la retroalimentación efectiva para la autoeficacia debe incluir espacio para debates en clases acerca de las soluciones adecuadas e inadecuadas para los problemas de traducción (Dollerup, 1994). Por otra parte, la retroalimentación debe ser comunicada en entornos de aprendizaje en donde la capacidad para traducir se vea como una habilidad que se puede adquirir y mejorar (Torre, 2007), lo cual acá no sucede dado que el hecho de que la profesora genere que todos se queden en silencio, que no tome en cuenta las versiones traducidas por sus estudiantes $y$, «tenga que ser esta palabra y no hay más", da cuenta de un ambiente tenso, estricto y muy poco constructivo.

\footnotetext{
2 Dicho participante emitió múltiples citas acerca de retroalimentación severa, pero solo se optaron por esas dos debido a limitaciones de longitud del artículo.
}

Hikma 19 (2) (2020), 291 - 326 
Lo expresado anteriormente se ve reflejado en el siguiente discurso acerca de retroalimentación impositiva3:

Es que a veces se ha enojado la profesora y empieza a reclamar que uno tiene que traer las cosas hechas para no discutirlas en clase, entonces quedan todos en silencio. Entonces ahora se tiene que hacer así o así, no hay mucha flexibilidad a cómo se hace. Eso yo creo que es uno de los grandes problemas. (Entrevista 2, p. 11)

\subsection{Matriz Compañeros de curso}

La matriz de Compañeros de curso (Figura 2) se compone de discursos que aluden a retroalimentación y a trabajo en equipo.

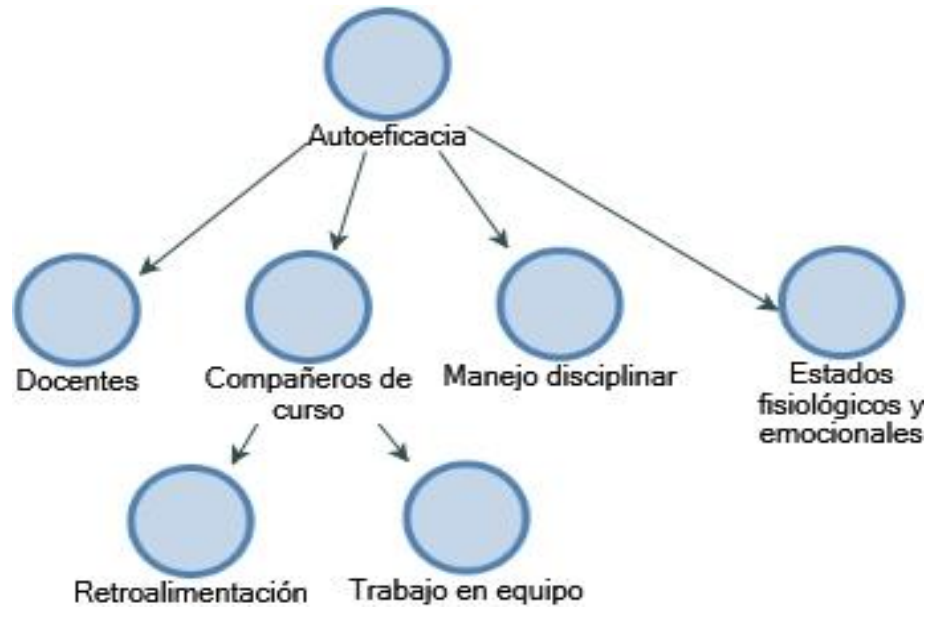

Figura 2. Matriz "Compañeros de curso». Fuente: Elaboración propia

\subsubsection{Retroalimentación}

Entre los discursos de los entrevistados, se evidenció que la retroalimentación emitida por sus compañeros de curso actuaba como un factor de influencia positiva en su autoeficacia. Pues, dentro de la disciplina de la Traducción, la retroalimentación o feedback constructivo, además de los docentes, también puede ser elaborada por los compañeros de curso (Alfayyadh, 2016; Bolaños-Medina y Núñez, 2018; Haro-Soler, 2017). Asimismo, según Alfayyadh (2016), los estudiantes suelen considerar los comentarios de sus compañeros menos intimidantes que los del profesor, por lo tanto, pueden ser más fáciles de aceptar. Tal como se mencionó en

${ }^{3}$ Dos de los tres entrevistados emitieron discursos acerca de retroalimentación positiva y por temas de limitaciones de longitud del artículo se optó solo por esta cita para ejemplificar

Hikma 19 (2) (2020), 291 - 326 
apartados anteriores, la persuasión verbal en forma de retroalimentación puede reforzar o disminuir la autoeficacia (Prieto, 2007; Zalazar, Aparicio, Ramírez, Garrido, 2011). Sin embargo, en el caso de todos los discursos citados, la retroalimentación por parte de sus pares ha sido algo beneficioso para el incremento de la autoeficacia.

Lo anterior se ve reflejado en los siguientes discursos que aluden a la retroalimentación emitida por compañeros de curso ${ }^{4}$ :

Está el feedback de nuestros compañeros. A veces, nos intercambiamos los trabajos o traducciones y nos evaluamos: «en esta parte, fíjate», por ejemplo. Porque uno a veces está muy metido y lo ha visto tantas veces, que no ve el error. Entonces, es mejor que otra persona, que quizás no haya estado tan pendiente, con otra perspectiva, te diga los errores. Eso ha sido una gran ayuda. (Entrevista 1, p. 7)

Entrevistadora: ¿Tú sientes que ese feedback más colaborativo entre compañeros ayuda?, ¿es positivo?

Entrevistado: Sí, porque nos dan opciones que, quizás, nunca se nos hubieran ocurrido. Por ejemplo, en un párrafo tuve tal o tal problema, escucho al resto y siempre voy anotando: usar estos ejemplos, esta estructura, poner una metáfora, no sé. (Entrevista 1 , p. 8).

\subsubsection{Trabajo en equipo}

Los participantes se refirieron a una especie de confianza generada a través del trabajo colaborativo con sus compañeros de curso. Lo anterior es algo muy positivo (Haro-Soler, 2019), debido a que el trabajo colaborativo entre traductores produce múltiples perspectivas ante temas problemáticos (Kiraly, 2000), ayuda a desarrollar la autonomía de los estudiantes (Carabajal, 2017), los empodera para enfrentar desafíos, crear experiencias de aprendizaje significativas, mejorar su competencia de traducción (GalánMañas, 2011; Kiraly, 2000, 2012) y favorecer la confianza en cuanto a la toma y la justificación de decisiones (Haro-Soler, 2019), lo cual puede transformase en experiencias directas, una fuente principal de autoeficacia (Bandura, 1997; Pajares, 1997; Woolfolk, 2014).

\footnotetext{
4 Todos los entrevistados emitieron discursos acerca de retroalimentación emitida por los compañeros de curso. Sin embargo, por temas de limitaciones de longitud del artículo se optaron solo por estas dos citas para ejemplificar.
}

Hikma 19 (2) (2020), 291 - 326 
A continuación, se presentan algunos de los discursos que aluden a trabajo en equipo y su influencia positiva en la autoeficacia ${ }^{5}$ :

Lo que tengo entendido, es que la traducción en sí, es un trabajo en equipo, en las empresas de traducción siempre hay un trabajo en equipo. Entonces, el hecho de saber que uno no está solo en el proceso, o, también, como estudiantes, como veo a mis compañeros que están en las mismas. Entonces, saber que no estoy solo en este proceso, sino que, también, hay otras personas que están en lo mismo, que pueden tener los mismos problemas o que me pueden ayudar a resolver ciertos temas o dificultades. (Entrevista 1, p. 8).

Entrevistadora: ¿Qué momentos o situaciones o personas 0 factores crees que han influido positivamente en tú confianza?

Entrevistado: Cuando empezamos a trabajar más en equipo, porque empezamos a determinar cada proceso por persona, cada etapa de la traducción y en ese grupo empezamos a tener mucha confianza, en todo ámbito, nos hicimos muy amigos, somos colegas y a la vez amigos, eso generó mucha confianza entre todos nosotros, superamos las expectativas que nosotros mismos nos habíamos puesto, entonces eso sirvió mucho. (Entrevista 3, p. 8).

\subsection{Matriz de Manejo disciplinar}

La matriz de Manejo disciplinar (Figura 3), en relación con la autoeficacia, apunta a que mientras más manejo disciplinar se tiene de una cierta área temática, más confianza tenían los entrevistados para traducir. Dicho manejo disciplinar, tanto en presencia como ausencia, provenía de la formación recibida en la universidad, documentación, interés personal y ausencia de experiencia previa. Por motivos teóricos, los temas de formación, documentación y ausencia de experiencia previa serán analizados en conjunto.

\footnotetext{
${ }^{5}$ Todos los entrevistados emitieron discursos acerca de trabajo en equipo. Sin embargo, por temas de limitaciones de longitud del artículo se optaron solo por estas dos citas para ejemplificar.
}

Hikma 19 (2) (2020), 291 - 326 


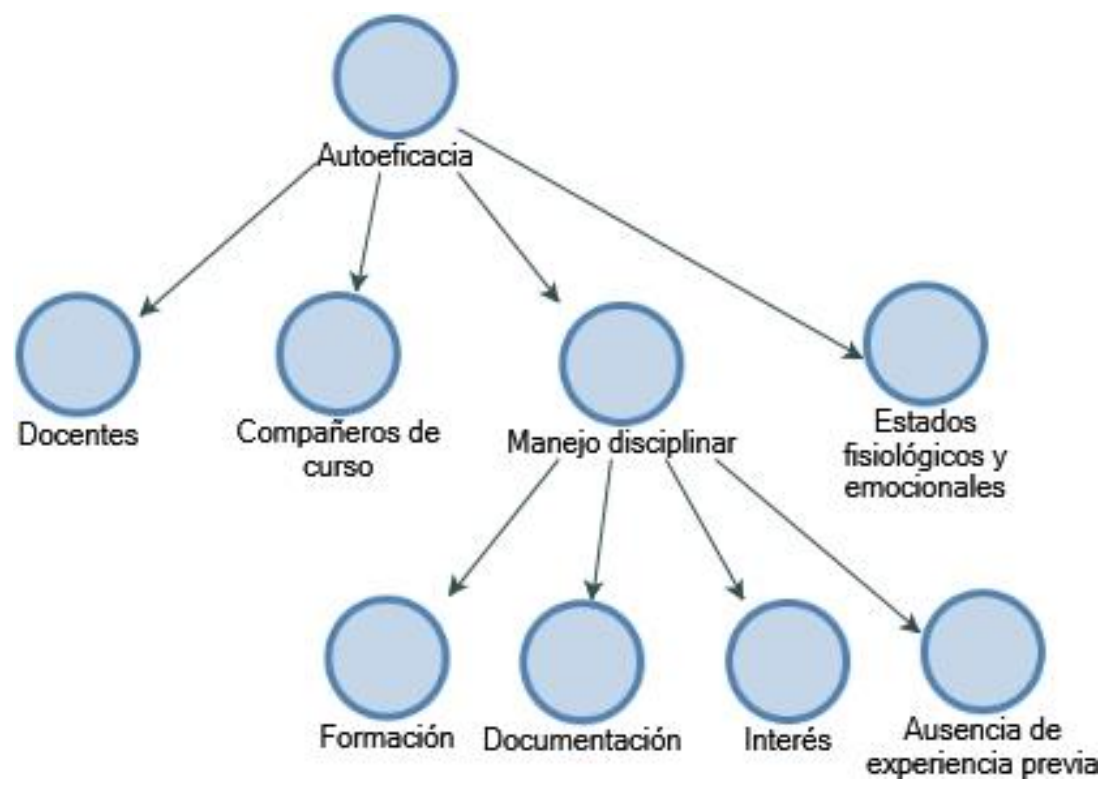

Figura 3. Matriz «Manejo disciplinar». Fuente: Elaboración propia

\subsubsection{Formación, documentación y ausencia de experiencia previa}

Según lo expresado por los participantes, los estudiantes se sienten más confiados para traducir aquellos temas en los que han tenido formación previa por parte de su universidad, también se sienten más confiados cuando se han documentado previamente y, asimismo, con temáticas que ya han traducido antes.

Estos tres factores (formación, documentación y experiencia previa) hacen referencia a una de las cuatro fuentes principales de autoeficacia: las experiencias directas o de dominio. Así pues, el completar exitosamente una tarea o actividad incrementa la autoeficacia (Bandura, 1997; Pajares, 1997; Woolfolk, 2014). Sin embargo, en el caso de los participantes, sucedía lo contrario dado que no contaban con dichas experiencias de dominio cuando se trató del texto utilizado en la etapa de protocolo verbal retrospectivo. Esto está relacionado con el hecho de que tener una autoeficacia deficiente hace que las personas consideren las tareas que deben realizar como más complejas de lo que realmente son (Bandura, 1997; Cabanach et al., 2010), pues los entrevistados manifestaron sentirse «intimidados» e intranquilos ante un área temática en la que no habían tenido experiencia, ni formación ni documentación previa. 
Asimismo, según Bandura (1997), la autoeficacia también influye en las actividades que los individuos deciden emprender dado que las personas suelen evitar aquellas actividades para las que no se sienten capacitados y esto se evidenció entre los entrevistados dado que uno de ellos, cuando se refirió al texto aplicado en la etapa de protocolo verbal retrospectivo «prefiero confiárselo a otra persona» debido a su falta de documentación y experiencia.

Por su parte, la documentación no solo es importante porque, en el caso de los estudiantes de Traducción, influye en la autoeficacia, sino que también es relevante porque se incluye en más de un modelo de la competencia traductora (Grupo PACTE, 2001; Kelly, 2002), es decir, se considera una habilidad necesaria para poder ejercer la actividad traslativa de forma profesional.

Lo anterior se ve reflejado en los siguientes discursos que aluden a formación, la autoeficacia:

\subsubsection{Formación}

El siguiente es uno de los discursos que aluden a formación, documentación y ausencia de experiencia previa 6 :

Entrevistadora: ¿Tú crees que tener un espectro más variado, más amplio de temas dan más confianza?

Entrevistado: Sí, de todas maneras. (Entrevista 2, p. 12)

\subsubsection{Documentación}

Los siguientes son algunos de los discursos que aluden a documentación $n^{7}$ :

Hoy en día me siento más seguro, sin embargo, cuando se presenta un tema que no manejo mucho, se genera un miedo. (Entrevista 3 , p. 13)

Entrevistadora: ¿Qué crees tú que te hubiese ayudado a sentirte con más confianza con respecto a tu propia traducción en este caso?

Entrevistada: De todas maneras saber anteriormente de qué se va a tratar la traducción para yo poder investigar, leer, ver cosas en internet de qué se trata esto, buscar los términos, tenerlos antes y haber dicho... estar correctamente que estos términos, hacer mi propio glosario, sabiendo lo que vamos a hablar, para decir esto es

\footnotetext{
${ }^{6}$ Todos los entrevistados emitieron discursos acerca de formación. Sin embargo, por temas de limitaciones de longitud del artículo, se optaron solo por estas dos citas para ejemplificar.

${ }^{7}$ Todos los entrevistados emitieron discursos acerca de documentación. Sin embargo, por temas de limitaciones de longitud del artículo, se optaron solo por estas dos citas para ejemplificar.
}

Hikma 19 (2) (2020), 291 - 326 
así y no hay equivocación en los términos, no tener dudas. Saber $100 \%$ que esto es esto y no hay equivocación. (Entrevista 2, p. 6)

\subsubsection{Ausencia de experiencia previa}

Los siguientes discursos aluden a ausencia de experiencia previa ${ }^{8}$ :

Bueno, primero que todo, la medicina en sí, creo que nunca había traducido un texto de medicina, por lo que el léxico utilizado es bastante desconocido para mí. Principalmente, fue el equivalente de los términos, el mayor problema y, también, el hecho de que no estoy acostumbrada a traducir desde el español al inglés, sino que del inglés al español. (Entrevista 1, p. 1)

Al principio me sentí un poco incómodo con la traducción, porque tenía una terminología compleja, que yo nunca había tratado antes. (Entrevista 3, p. 1)

\subsubsection{Interés}

La traducción es un proceso que involucra factores afectivos y actitudinales (Laukkanen, 1996; Tirkkonen-Condit y Laukkanen, 1996) y está mediado por la motivación (Darwish, 2010; Jääskeläinen, 1996). Asimismo, Mathur y Chandran (2014) aseguran que la mayoría de los traductores son profesionales impulsados por una pasión por su trabajo. Estos factores vocacionales y motivacionales, junto con la afinidad personal del traductor, afectan positivamente la calidad de la traducción (Malmkjær, 2009). Por ende, es natural que se sientan más confiados para traducir en aquellas áreas por las que tienen mayor interés, pues se sienten más motivados para documentarse e investigar de dichos temas, lo cual se traduce en una experiencia previa, y subsecuentemente, en una experiencia de dominio (Bandura, 1997), lo cual es una fuente principal de autoeficacia.

Lo anterior se ve reflejado el siguiente discurso que alude a interés 9 :

Entrevistadora: En tú caso ¿hay ciertas áreas en las que te sientas más preparado o más confiado y capaz que otras?

Entrevistado: Sí, el área humanista más que todo, me gusta mucho el tema de la política, lo social, lo medioambiental.

Entrevistadora: $Y$ ¿por qué te sientes particularmente con más confianza en esas áreas?

\footnotetext{
8 Todos los entrevistados emitieron discursos acerca de ausencia de experiencia previa. Sin embargo, por temas de limitaciones de longitud del artículo, se optaron solo por estas dos citas para ejemplificar.

9 Todos los entrevistados emitieron discursos acerca de interés. Sin embargo, por temas de limitaciones de longitud del artículo, se optaron solo por estas dos citas para ejemplificar.
}

Hikma 19 (2) (2020), 291 - 326 
Entrevistado: Porque en realidad siempre desde niño, he sido muy apegado al medioambiente y desde que entré a la adolescencia empecé a leer mucho de política, problemas sociales, que me fui dando cuenta que de alguna forma te afectan, no de forma directa, y poco a poco me fui interesando en esos temas, introduciéndome más allá de lo que está a simple vista.

Entrevistadora: Entonces ¿crees que en tú caso, mientras más te guste un tema, más confiado te sientes para traducirlo?

Entrevistado: Sí, totalmente. (Entrevista 3, p. 5)

\subsection{Matriz Estados fisiológicos y emocionales}

La matriz de Estados fisiológicos y emocionales (Figura 4) se configura de discursos que aluden al cansancio físico, el estado emocional y ergonomía. Por motivos teóricos, los temas de cansancio físico y estado emocional serán analizados en conjunto.

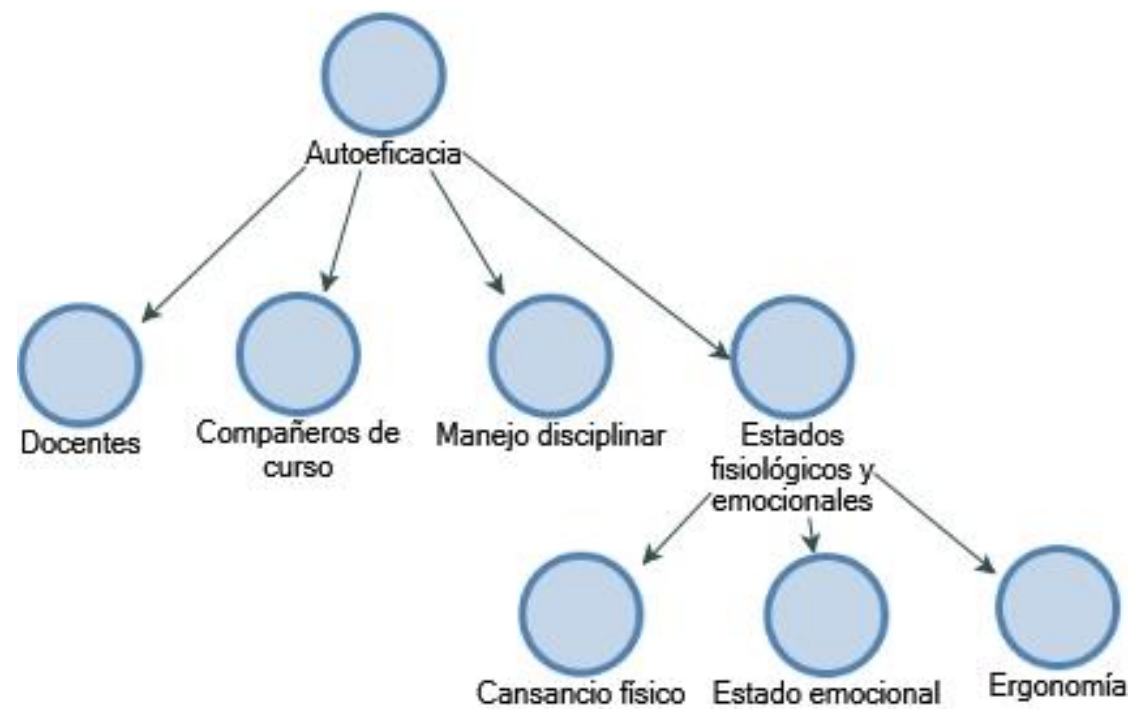

Figura 4. Matriz «Estados fisiológicos y emocionales». Fuente: Elaboración propia

\subsubsection{Cansancio físico y estado emocional}

Los estados fisiológicos y emocionales corresponden a una fuente principal de autoeficacia (Bandura, 1997). Esto es, los estados de ánimo y sentimientos positivos y negativos que las personas experimentan durante una actividad (Bandura, 1997) actúan como activadores positivos o negativos

Hikma 19 (2) (2020), 291 - 326 
de la autoeficacia, lo cual resulta en el sentimiento de estar o no «mentalmente preparado» (Woolfolk, 2014). Si lo extrapolamos a los discursos de los entrevistados, los sentimientos negativos como el cansancio, estrés, somnolencia y angustia disminuyen la autoeficacia (Usher y Pajares, 2009; Zalazar, Aparicio, Ramírez, Garrido, 2011)). Por ende, para que los estudiantes de traducción se sientan más preparados para traducir, no basta con que se documenten, hayan tenido formación o experiencia previa, sino que deben cuidar de su salud, tanto emocional como física.

Lo anterior se ve reflejado en los siguientes discursos que son algunos de los que aluden a cansancio físico y estados emocionales ${ }^{10}$ :

Entrevistadora: Y si lo llevamos a este texto que acabas de traducir ¿qué factores crees que influyeron de buena forma para sentirte más confiado y qué factores influyeron de mala forma?

Entrevistado: De mala forma, el tema que he dormido poco, el cansancio, la falta de concentración y en general he tenido una semana agitada. (Entrevista 3, p. 11)

Entrevistadora: ¿Crees que un traductor que está con su estado emocional adecuado se le hace más cómodo traducir que uno que no está en su mejor momento emocionalmente?

Entrevistado: Claro que sí, creo que en todo ámbito, por un momento uno puede aguantar todo eso y traducir, pero llega un momento en que igual te puede colapsar. (Entrevista 3, p. 11)

\subsubsection{Ergonomía}

Si bien, la ergonomía como tal no está considerada como una fuente de autoeficacia dentro de la teoría cognitivo-social, teniendo en cuenta los discursos de los entrevistados, se puede apreciar que, en el caso de los traductores, guarda cierta relación con una de las cuatro fuentes principales de autoeficacia: los estados emocionales y psicofisiológicos (Bandura, 1997). El no contar con un ambiente ergonómicamente apto produce estados fisiológicos y emocionales negativos, los cuales se traducen en detrimento de la autoeficacia. Debido a dada la gran cantidad de tiempo que los traductores pasan sentados frente a un computador, pueden ser víctimas de múltiples enfermedades tanto físicas como psicológicas, por ejemplo, problemas musculoesqueléticos, afecciones en los ojos, cuello y cintura escapular, cefaleas, estrés, síndrome del túnel carpiano, tendinitis, dolor de espalda,

\footnotetext{
10 Dos de los tres entrevistados emitieron discursos acerca de cansancio físico y estados emocionales. Sin embargo, por temas de limitaciones de longitud del artículo, se optaron solo por estas dos citas para ejemplificar.
}

Hikma 19 (2) (2020), 291 - 326 
fatiga visual, obesidad, enfermedades gastrointestinales, entre otras. (Ferreira y Schwieter, 2015; Rodríguez, 2016).

Asimismo, según Van Egdom, Segers, Kokaert y Cadwell (2018), para que los estudiantes de Traducción puedan enfrentar la presión, el estrés y la competencia en el futuro y para sepan satisfacer las necesidades de la industria, se deben desarrollar, enseñar y adoptar prácticas ergonómicas sostenibles dentro del aula. Por ende, no es un factor que podemos obviar cuando se habla de autoeficacia en estudiantes de traducción.

Lo anterior se ve reflejado en el siguiente ejemplo de discurso que alude a ergonomía ${ }^{11}$ :

Entrevistadora: En general cuando traduces ¿cómo te sientes, cómodo, incómodo, tienes confianza en ti mismo?

Entrevistado: Sí, pero igual yo creo que eso se desarrolla en un buen ambiente, una silla buena, un escritorio adecuado. Cuando el texto es muy largo y tienes que estar horas traduciendo y editando yo creo que un buen lugar de trabajo es muy necesario. El silencio, todo. (Entrevista 3, p. 5)

\section{CONCLUSIONES}

Finalmente, podemos afirmar que, en cuanto a la Escala de Autoeficacia General, a pesar de su frecuente uso en el campo de los estudios de Traducción, presenta muy poca especificidad para medir la autoeficacia en estudiantes de Traducción y su grado de confiabilidad es muy bajo dado que arroja resultados extremadamente homogéneos. Por esta razón, dado que esta es la primera vez que se aplica a estudiantes de traducción en Chile, como precedente para futuras investigaciones, sería conveniente preferir un instrumento específico para medir la autoeficacia en traducción, como la «La Escala de Creencias de Autoeficacia del Traductor», creada por Haro-Soler (2018a).

En cuanto a los factores que influyen en la autoeficacia de los estudiantes de Traducción se encuentran: los profesores, los compañeros de curso, el manejo disciplinar que tengan los estudiantes y sus estados fisiológicos y emocionales.

Con respecto al rol de los profesores y su influencia en la autoeficacia, esto se encuentra en consonancia con el estudio desarrollado por Haro-Soler (2018b) en España. La retroalimentación que estos proporcionan es decisiva, por lo tanto, debería ser un elemento relevante que los docentes de

\footnotetext{
11 Uno de los tres entrevistados emitió múltiples citas acerca de ergonomía. Sin embargo, por temas de limitaciones de longitud del artículo, se optaron solo por esa cita para ejemplificar
} 
Traducción estuviesen siempre capacitados para entregar retroalimentación de forma adecuada y tener mucho cuidado con las retroalimentaciones severas o impositivas dado que actúan en desmedro directo de la autoeficacia de sus estudiantes.

En cuanto a los compañeros de curso, el trabajo en equipo y la retroalimentación entre pares corresponde a un factor de influencia altamente positiva en la autoeficacia del estudiantado, por lo tanto, es favorable propiciar climas de aula en donde prime el trabajo colaborativo. Siendo este último también un elemento común encontrado en un estudio español de Haro-Soler (2019).

Dado que el manejo disciplinar también corresponde a un factor de influencia en la autoeficacia, los programas de traducción deberían abordar un amplio espectro de temáticas de traducción, pues mientras más manejo y experiencia previa tengan en un área del conocimiento en particular, más confiados se sienten los estudiantes para traducir. Asimismo, los programas de Traducción deben preparar a sus alumnos para poder realizar un buen proceso de documentación previa dado que tiene bastante injerencia en el manejo disciplinar. Por otro lado, dichos programas también deberían tener en cuenta y desarrollar los intereses personales de sus estudiantes dado que, se sienten con más confianza para traducir aquellas áreas temáticas hacia las que sienten mayor afinidad.

En lo que concierne a sus estados fisiológicos y emocionales, la salud física y emocional de los estudiantes influye tanto de manera positiva como negativa en la autoeficacia. Por consiguiente, debería ser un factor de atención para las instituciones que imparten la carrera, tanto de resguardo y cuidado emocional como salas de clase ergonómicamente adecuadas específicamente para traductores.

Un hallazgo muy peculiar que se evidenció fue que, en cuanto a las experiencias vicarias, al contrario de lo que se esperaría según lo estipulado por la teoría cognitivo-social y los grados de similitud (Bandura, 1995), y si lo comparamos con el estudio desarrollado por Haro-Soler (2018b), no se manifestaron experiencias vicarias a través compañeros de curso o exalumnos de la carrera, sino por medio de los docentes. Aunque esto podría deberse a que los profesores aludidos en las entrevistas eran muy propensos a compartir anécdotas laborales y de sus días estudiantiles.

Finalmente, los aportes de este estudio se orientan a proporcionar información pionera en Chile sobre medición de autoeficacia en estudiantes de Traducción, constatando que la Escala de Autoeficacia General no discrimina y arroja resultados muy homogéneos. Asimismo, buscan generar aportes teóricos y sentar precedentes para futuras investigaciones dado que 
es la primera vez que se estudia la autoeficacia en estudiantes de traducción en Chile. Asimismo, dado que, en el contexto nacional, la traducción profesional es una carrera con bajo ingreso monetario, poca valoración social y baja empleabilidad, debemos fomentar la autoeficacia en nuestros estudiantes dado que necesitarán confiar en sí mismos para poder desempeñarse profesionalmente en un contexto tan adverso.

\section{REFERENCIAS}

Albin, J. (2012). Competencia y autoeficacia: Estudio de los factores afectivos en el traductor. Hikma, 11(1), 9-33. doi: https://doi.org/10.21071/hikma.v11i.5242

Alexieva, B. (1990). Creativity in simultaneous interpretation. Babel, 36(1), 1 6. doi: https://doi.org/10.1075/babel.36.1.02ale

Alfayyadh, H. (2016). The feedback culture in translator education: a comparative exploration of two distinct university translation programs (Tesis Doctoral). Kent State University. Ohio, Estados Unidos.

Al-Hassan, A. (2013). The Importance of Culture in Translation: Should Culture Be Translated? International Journal of Applied Linguistics \& English Literature, 2(2), 96-100. doi: http://dx.doi.org/10.7575/aiac.ijalel.v.2n.2p.96.

Araghian, R., Ghonsooly, B. y Ghanizadeh, A. (2018). Investigating problemsolving strategies of translation trainees with high and low levels of selfefficacy. Translation, cognition and behaviour, 1(1), 74-97. doi: https://doi.org/10.1075/tcb.00004.ara

Aragón, R. (2012). Teoría Cognitivo-Social de Albert Bandura. España: Psiqueviva. Recuperado de https://psiqueviva.com/teoria-cognitivosocial-de-albert-bandura/

Atkinson, D. (2012). Freelance translator success and psychological skill: a study of translatro competece with perspectives from wotk psychology (Tesis Doctoral). University of Auckland. Auckland, Nueva Zelanda.

Atkinson, D. y Crezee, I. (2014). Improving Psychological Skill in Trainee Interpreters. International Journal of Interpreter Education, 6(1), 3-18. doi: https://doi.org/10.1075/ts.3.01atk

Ballard, M. (1997). Créativité et traduction. Target. 9(1), 85-110. doi: https://doi.org/10.1075/target.9.1.06bal

Bandura, A. (1986). Social foundations of thought and action: A social cognitive theory. Englewood Cliffs, New Jersey: Prentice Hall. 
Bandura, A. (1987). Pensamiento y acción: Fundamentos sociales. Barcelona, España: Martínez Roca.

Bandura, A. (1989). Human Agency in Social Cognitive Theory. American Psychologist, 44(9), 1175-1184. doi: https://doi.org/10.1037/0003066X.44.9.1175

Bandura, A. (1994). Self-efficacy. En Vilayanur S. Ramachaudran (Ed.), Encyclopedia of Human Behavior (pp. 71-81). Nueva York, Estados Unidos: Academic Press.

Bandura, A. (1995). Self-Efficacy in Changing Societies. Cambridge, Inglaterra: Cambridge University Press.

Bandura, A. (1997). Self-Efficacy: The Exercise of Control. Nueva York, Estados Unidos: Worth Publishers.

Bernasconi, A. (2004). Informe sobre la educación superior en Chile, 19802003. Santiago, Chile: Editorial Universitaria

Blanco Vega, H., Martínez Martín, M., Zueck Enríquez, M. C. y Gastélum Cuadras, G. (2011). Análisis psicométrico de la escala de autoeficacia en conductas académicas en universitarios de primer ingreso. Actualidades Investigativas en Educación, 11(3), 1-27. doi: https://doi.org/10.15517/aie.v11i3.10214

Bolaños-Medina, A. (2014). Self-efficacy in translation. Translation and Interpreting Studies, 9(2), 197-218. doi: https://doi.org/10.1075/tis.9.2.03bol

Bolaños-Medina, A. (2015). La tolerancia a la ambigüedad y los procesos cognitivos del traductor. Babel, 61(2), 147-169. doi: https://doi.org/10.1075/babel.61.2.01bol

Bolaños-Medina, A. y Núñez, J. (2018). A Preliminary Scale for Assessing Translators' Self- efficacy. Across Languages and Cultures, 19(1), 5378. doi: https://doi.org/10.1556/084.2018.19.1.3

Brookhart, S. M. (2008). How to give effective feedback to your students. Virginia, Estados Unidos: ASCD.

Cabanach González, R. G., Valle, A., Rodríguez Martínez, S., Piñeiro, I. y González Millán, R. (2010). Las creencias motivacionales como factor protector del estrés en estudiantes universitarios. European Journal of Education and Psychology, 3(1), 75-87. doi: https://doi.org/10.30552/ejep.v3i1.49 
Canto y Rodríguez, J. (1998). Autoeficacia y educación. Revista Educación y Ciencia, 2(18), 45-53. Recuperado de http://www.educacionyciencia.org/index.php/educacionyciencia/article/ view/142

Carabajal, R. (2017). Propuesta didáctica para abordar la traducción técnica: trabajo colaborativo y Aprendizaje Basado en Proyectos. Revista Digital De Investigación En Docencia Universitaria, 11(2), 192-202. doi: http://dx.doi.org/10.19083/ridu.11.571

Charness, N. y Ericsson, K. (1997). Cognitive and Developmental Factors in Expert Performance. En P. Feltovich, K. Ford y R. Hoffman (Eds.), Expertise in Context: Human and Machine (pp.3-41). Cambridge, Inglaterra: MIT Press.

Collombat, I. (2016). Doubt and negotiation: The perception of professional translators. Meta, 61(1), 145-164. doi: https://doi.org/10.7202/1036987ar

Comisión Nacional de Acreditación (2020). Acreditación Institucional. Recuperado de https://www.cnachile.cl/Paginas/Acreditacioninstitucional.aspx

Darwish, A. (2010). Translation Applied!: An Introduction to Applied Translation Studies - A Transactional Model. Chelsea Heights, VIC, Australia: Writescope Pty Ltd

Denzin, N. y Lincoln, Y. (2002). The Qualitative Inquiry Reader, Estados Unidos: Sage Publications.

Dollerup, C. (1994). Systematic feedback in teaching translation. En C. Dollerup \& A. Lindegaard (Eds.), Teaching Translation and Interpreting 2: Insights, Aims, Visions (pp. 121-132). Amsterdam y Philadelphia: John Benjamins.

Ferreira, A. y Schwieter, J. (2015). The Handbook of Translation and Cognition. West Sussex, Inglaterra: Wiley-Blackwell.

Fox, O. (2000). The use of translation diaries in a process-oriented translation teaching methodology. En C. Schaeffner y B. Adab (Eds.). Developing translation competence (pp. 115-130). Amsterdam/Filadelphia: John Benjamin.

Fraser, J. (1995). Professional versus student behaviour. En Cay Dollerup y Vibeke Appel (Eds). Teaching Translation and Interpreting 3: New Horizons. Amsterdam: John Benjamins. 243-250. 
Froeliger, N. (2004). Les mécanismes de la confiance en traduction: aspects relationnels [Mechanism of confidence in translation: Related aspects]. The Journal of Specialized Translation, 2(1), 24-39. doi: $10.4000 /$ traduire.975

Galán-Mañas, A. (2011). Translating authentic technical documents in specialised translation classes. The Journal of Specialised Translation, 16(1), 109-125. Recuperado de https://www.researchgate.net/publication/308416761_Translating_aut hentic_technical_documents_in_specialised_translation_classes

González, M. C. y Tourón, J. (1992). Autoconcepto y rendimiento escolar: Sus implicaciones en la motivación y en la autorregulación del aprendizaje. Pamplona, España: Ediciones de la Universidad de Navarra.

Gonzalez-Davies, M. y Scott-Tennet, C. (2005). A Problem-Solving and Student-Centred Approach to the Translation of Cultural References. Meta, 50(1), 160-179. doi: https://doi.org/10.7202/010666ar

Göpferich, S. (2009). Towards a model of translation competence and its acquisition: the longitudinal study TransComp. En Göpferich, S., Jakobsen, A. y Mees, I. (Eds.). Behind the mind: methods, models and results in translation process research (pp. 12-37). Copenhague, Dinamarca: Samfundslitteratur.

Grupo PACTE. (2001). La competencia traductora y su adquisición. Quaderns: Revista de traducció, 6(1), 39-45. Recuperado de https://ddd.uab.cat/pub/quaderns/11385790n6/11385790n6p39.pdf

Hamui-Sutton, A. (2013). Un acercamiento a los métodos mixtos de investigación en educación médica. Revista Investigación en Educación Médica, 2(8), 211-216. doi: https://doi.org/10.1016/S20075057(13)72714-5

Hansen, G. (2003). Controlling the Process. Theoretical and Methodological Reflections on Research into Translation Processes. En F. Alves (Ed.) Triangulating Translation (pp.25-42). Amsterdam/Philadelphia: John Benjamins.

Hansen, G. (2005). Experience and Emotion in Empirical Translation Research with Think-Aloud and Retrospection. Meta, 50(2), 511-521. doi: https://doi.org/10.7202/010997ar

Haro-Soler, M. M. (2017). ¿Cómo desarrollar la autoeficacia del estudiantado? Presentación y evaluación de una experiencia formativa 
en el aula de traducción. Revista Digital de Docencia Universitaria. 11(2), 50-74. doi: http://dx.doi.org/10.19083/ridu.11.567

Haro-Soler, M. M. (2018a). Las creencias de autoeficacia del estudiantado de traducción: una radiografía de su desarrollo. (Tesis Doctoral). Universidad de Granada, Departamento de Traducción e Interpretación. España

Haro-Soler, M.M. (2018b). Self-confidence and its role in translator training the students' perspective. En Jääskeläinen, R. y Lacruz, I. (Ed.) Innovation and Expansion in Translation Process Research. (pp. 131160).

Haro-Soler, M.M. (2019). La colaboración entre futuros traductores: su impacto en las creencias de autoeficacia para traducir. Mutatis Mutandis 12(2), 330-356. doi: 10.17533/udea.mut.v12n2a01

Hattie, J. y Timperley, H. (2007). The power of feedback. Review of Educational Research, 77(1), 81-112. doi: https://doi.org/10.3102/003465430298487

Ho, H. (2010). The Relationship between Levels of Expertise, Task Difficulty, Perceived Self-efficacy, and Mental Effort Investment in Task Performance (Tesis Doctoral). University of Southern California, California.

Hurtado Albir, A. (2001). Traducción y traductología: introducción a la traductología. Madrid, España: Cátedra.

Huxham, M. (2007). Fast and effective feedback: are model answers the answer? Assessment and Evaluation in Higher Education, 32(6), 601611. doi: https://doi.org/10.1080/02602930601116946

Jääskeläinen, R. (1996). Hard work will bear beautiful fruit: A comparison of two think-aloud protocol studies. Meta, 41(1), 60-74. doi: https://doi.org/10.7202/003235ar

Jiménez, A., Ruiz, M. y Pinazo, D. (2014). Self-efficacy and language proficiency in interpreter trainees. The Interpreter and Translator Trainer, 8(2), 167-182. doi: https://doi.org/10.1080/1750399X.2014.908552

Kelly, D. (2002). Un modelo de competencia traductora: bases para el diseño curricular. Puentes, 1(1), 9-20. Recuperado de https://www.researchgate.net/publication/237538245_Un_modelo_de _competencia_traductora_Bases_para_el_diseno_curricular 
Kiraly, D. (1995). Pathways to translation. Pedagogy and process. Kent, Ohio: Kent State University Press.

Kiraly, D. (2000) A Social Constructivist Approach to Translator Education; Empowerment from Theory to Practice. Manchester, UK; Northampton, MA: St. Jerome Publishing.

Kiraly, D. (2012). Growing a project-based translation pedagogy: A fractual perspective. Meta: Translators' Journal, 57(1), 82-95. doi: https://doi.org/10.7202/1012742ar

Krueger, N. y Dickson, P. (1994). How Believing in Ourselves Increases Risk Taking: Perceived Self-efficacy and Opportunity Recognition. Decision Sciences, 25(3), 385-400. doi: 10.1111/j.1540-5915.1994.tb00810.x

Kussmaul, P. (1995). Training the Translator. Amsterdam: Benjamin.

Laukkanen, J. (1996). Affective and Attitudinal Factors in Translation Processes. Target. International Journal of Translation Studies, 8(2), 257 -274. doi: https://doi.org/10.1075/target.8.2.04lau

Lorenzo, M. P. (1999). La seguridad del traductor profesional en la traducción a una lengua extranjera. En Hansen, G. (Ed.) Probing the process in translation: methods and results (pp. 121-134). Copenhague: Samfundslitteratur.

Lorenzo, M. P. (2002). ¿Es posible la traducción inversa? Resultados de un experimento sobre traducción profesional a una lengua extranjera. Copenhagen studies in language, 27(1), 85-124. Recuperado de https://research.cbs.dk/en/publications/es-posible-latraducci\%C3\%B3n-inversa-resultados-de-un-experimento-sob

Maddux, E. (1995). Self-efficacy, adaptation, and adjustment. Theory, research, and application. (Ed.) Nueva York, Estados Unidos: Plenum Press.

Malmkjær, K. (2009). What is translation competence? Revue française de linguistique appliquée, 14(1), 121-134. doi : https://doi.org/10.3917/rfla.141.0121

Mashady, H., Fatollahi, M. y Pourgalavi, M. (2015). Self-efficacy and Prediction of Note-taking Inclination among Undergraduate Translation Students. Theory and Practice in Language Studies, 5(11), 2366-2372. doi: http://dx.doi.org/10.17507/tpls.0511.22

Mathur, S. y Chandran, M. (2014). Textual Travels: Theory and Practice of Translation in India. India: Routledge. 
Muñoz-Martin, R. (2009). Expertise and Environment in Translation. Mutatis Mutandis 2(1), 24-37. Recuperado de https://www.researchgate.net/publication/50863685_Expertise_and_E nvironment_in_Translation

Muñoz-Martín, R. (2014). Situating Translation Expertise: A review with a sketch of a construct. En J.W. SChwieter \& A. Ferreira (Ed.), The Development of Translation Competence: Theories and Methodologies from Psycholinguistics and Cognitive Science. (pp. 2-56). Inglaterra, Cambridge Scholars Publishing.

Naghi, M. (2000). Metodología de la Investigación. México: Limusa.

Nicol, D. J. y Macfarlane-Dick, D. (2006). Formative assessment and selfregulated learning: a model and seven principles of good feedback practice. Studies in Higher Education, 31(2), 199-218. doi: https://doi.org/10.1080/03075070600572090

Núñez, J. y Bolaños-Medina, A. (2017). Predictors of problem-solving in translation: implications for translator training. The Interpreter and Translator Trainer, 12(3), 282-298. doi: https://doi.org/10.1080/1750399X.2017.1359762

Pajares, F. (1997). Current directions in self-efficacy research. En Maehr, M. y Pintrich, P. (Eds.). Advances in motivation and achievement (pp. 149). Greenwich, CT: JAI Press.

Peinado, J. (2010). Efecto de la disciplina sobre la percepción de las competencias básicas en universitarios chihuahuenses (Tesis Doctoral). Universidad de Granada, España.

Pietrzak, P. (2014). Towards effective feedback to translation students: Empowering through group revision and evaluation. inTRAlinea. 1(1): Recuperado de http://www.intralinea.org/specials/article/towards_effe ctive_feedback_to_translation_students

Presas, M. (1998). Los componentes de la competencia pretraductora en el marco del diseño curricular. En I. García Izquierdo y J. Verdegal (Eds.). Estudios de traducción: un reto didáctico (pp. 131-134). Castelló de la Plana: Universitat Jaume I.

Prieto, L. (2007). Autoeficacia del profesor universitario: Eficacia percibida y práctica docente. Madrid, España: Narcea.

Pym, A. (1991). A Definition of Translational Competence, Applied to the Teaching of Translation. En Jovanovic, M. (Ed.), Translation: a creative 
profession $12^{\text {th }}$ world congress of FIT proceedings (pp. 541-546). Belgrado, Servia: Prevodilac.

Pym, A. (1992). Translation error analysis and the interface with language teaching. En Dollerup, C. y Loddegaard, A. (Eds). Teaching Translation and Interpreting. Training, Talent, and Experience (pp. 279-290). Amsterdam: John Benjamins.

Pym, A. (2003). Redefining translation competence in an electronic age: In defence of a minimalist approach. Meta, 48(4), 481-497. doi: https://doi.org/10.7202/008533ar

Rice, F. (1997). Desarrollo humano: estudio del ciclo vital. Nueva York, Estados Unidos: Pearson.

Rodríguez, M. (2016). Competencias y recursos para la práctica eficiente de la traducción profesional (PARTE II). Entreculturas 7(8), 259-277. Recuperado de https://dialnet.unirioja.es/descarga/articulo/5652303.p $\mathrm{df}$

Salanova Soria, M., Grau, R., Martínez Martínez, I., Cifre, E., Llorens, S. y García-Renedo, M. (2004). Nuevos horizontes en la investigación sobre la autoeficacia. Castelló, España: Publicacions de la Universitat Jaume I.

Scholz, U., Gutiérrez, B., Sud, S. y Schwarzer, R. (2002). Is General SelfEfficacy a Universal Construct? European Journal of Psychological Assessment, 18(3), 242-251. doi: https://doi.org/10.1027/10155759.18.3.242

Schwarzer, R. (1992). Self-efficacy: Thought Control of Action. Washington, DC: Hemisphere.

Tirkkonen-Condit, S. y Laukkanen, J. (1996). Evaluations: a Key Towards Understanding the Affective Dimension of Translational Decisions. Meta, 41(1), 45-59. doi: https://doi.org/10.7202/002360ar

Torre, J. (2007). Una triple alianza para un aprendizaje universitario de calidad. Madrid, España: Universidad Pontificia Comillas.

Usher, E. y Pajares, F. (2009). Sources of self-efficacy in mathematics: A validation study. Contemporary Educational Psychology, 34(1), 89-101. doi: https://doi.org/10.1016/j.cedpsych.2008.09.002

Valiante, G. (2000). Writing Self-efficacy and Gender Orientation: A developmental Perspective, A Dissertation Proposal. Atlanta, Georgia: Emory University. 
Van Egdom, G.-W., Segers, W., Kokaert, H., y Cadwell, P. (2018). Ergonomics in translator and interpreter training. The Interpreter and Translator Trainer, 12(2), 252-253. doi: https://doi.org/10.1080/1750399x.2018.1478451

Way, C. (2014). Structuring a legal translation course: a framework for decision-making in legal translator training. En King Kui Sin, L. y Wagner, A. (Eds.) The Ashgate handbook of legal translation (pp. 135152). Farnham: Ashgate.

Wilss, W. (1996). Knowledge and Skills in Translator Behavior. Amsterdam: John Benjamins.

Woolfolk, A. (2014). Psicología Educativa. México: Prentice Hall, Pearson.

Zalazar, M., Aparicio, M., Ramírez, C., y Garrido, S. (2011). Estudios preliminares de adaptación de la Escala de Fuentes de Autoeficacia para Matemáticas. Revista Argentina de Ciencias del Comportamiento, 3(2), 1-6. doi: https://doi.org/10.32348/1852.4206.v3.n2.5222

Zeldin, A. y Pajares, F. (2000). Against the Odds: Self-efficacy Beliefs of Women in Mathematical, Scientific, and Technological Careers. American Educational Research Journal, 37(1), 215-246. doi: https://doi.org/10.3102/00028312037001215 


\section{ANEXO 1. TEXTO UTILIZADO EN FASE DE PROTOCOLO VERBAL RETROSPECTIVO}

$\operatorname{vr}(a)$.

Presente

El examen realizado a su paciente ha dado el siguiente resultado:

RM Abdomen con Colangio-Resonancia

Antecedentes clinicos: Ictericia en estudio.

Hallazgos: La vesícula biliar se encuentra distendida, con un cálculo de $10 \mathrm{~mm}$ "atrapado" en el bacinete. La pared del bacinete se encuentra engrosada y adyacentes a un tejido con señal de partes blandas que se extiende por el conducto cístico e infiltra el hilio hepático, provocando una estenosis severa del conducto hepático común con leve a moderada dilatación de la vía biliar intrahepática. El tejido descrito mide aproximadamente $2,3 \mathrm{~cm}$ de diámetro mayor. Hay una segunda área nodular de engrosamiento de la pared vesicular en la cara inferior del cuerpo abarcando un área $3,8 \mathrm{~cm}$ con extensión al tejido adiposo perivesicular.

El hígado presenta forma y tamaño normal sin lesiones focales de aspecto agresivo en su parénquima. Vena porta principal y sus ramas intrahepáticas permeables al igual que las venas suprahepáticas, vena esplénica y vena mesentérica superior.

Tronco celíaco y sus ramas permeables, de calibre normal incluyendo arteria hepática propia y sus ramas intrahepáticas derecha e izquierda.

Páncreas de forma y tamaño normal identificando un quiste bilobulado de $15 \mathrm{~mm}$ en la región del cuerpo, sin caracteres tomográficos agresividad.

Glándulas suprarrenales y bazo de aspecto normal.

Riñones de forma y tamaño normal, sin hidronefrosis

No se identifican adenopatías retroperitoneales ni mesentéricas.

No hay ascitis.

En los segmentos visibles del tórax inferior se observa pequeña hernia gástrica hiatal por deslizamiento

Impresión diagnóstica: Colelitiasis 